\title{
SPORADIC HEMIPLEGIC MIGRAINE WITH PSYCHOTIC EPISODE AND MRI ABNORMALITIES - THERAPEUTIC DILEMMA: A CASE REPORT
}

\author{
Marina Mioč ${ }^{1}$, Vlatko Šulentić ${ }^{\text {, Ivan Jovanović }}{ }^{3}$, Vlasta Vuković-Cvetković ${ }^{4}$, \\ Sibila Nanković ${ }^{2}$ \& Željka Petelin Gadže ${ }^{2}$ \\ ${ }^{1}$ University Psychiatric Hospital Vrapče, University of Zagreb, Zagreb, Croatia \\ ${ }^{2}$ University Hospital Centre Zagreb, Department of Neurology, School of Medicine, University of Zagreb, \\ Referral Centre of the Ministry of Health of the Republic of Croatia for Epilepsy, Zagreb, Croatia \\ ${ }^{3}$ University Hospital Centre Zagreb, Department for Diagnostic and Interventional Radiology, \\ School of Medicine, University of Zagreb, Croatia \\ ${ }^{4}$ Danish Headache Center, Rigshospital - Glostrup, Copenhagen, Denmark
}

received: 2.7.2018;

revised: 31.10.2018;

accepted: 14.11.2018

$* * * * *$

\section{INTRODUCTION}

Hemiplegic migraine (HM) is a rare form of migraine with aura, classified as a complication of migraine in the ICHD 3 classification, which involves transient hemiparesis during the aura. There are two types of HM, sporadic hemiplegic migraine (SHM) with negative family history and familial hemiplegic migraine (FHM) where patient has at least one first- or second- degree relative with the same symptoms. FHM is genetically heterogeneous and dominantly inherited. Three FHM genes (CACNA1A, ATP1A2 and SCN1A) have been identified and some others are under investigation (Russell \& Ducros 2011, LaBianca et al. 2015). Attacks of hemiplegic migraine can present with various symptoms, rarely including fever, disturbance of consciousness and psychiatric changes (LaBianca et al. 2015). Differential diagnosis includes vasculitis, encephalitis, stroke, syndromes of mitochondrial encephalomyopathy, lactic acidosis, and stroke-like episodes (MELAS), cerebral autosomal dominant arteriopathy with subcortical infarcts and leukoencephalopathy (CADASIL), Creuzfeld-Jakob disease et cetera (Russell \& Ducros 2011, LaBianca et al. 2015). Diagnostic exams such as neuroimaging, electroencephalogram (EEG), cerebrospinal fluid (CSF) analysis are needed to exclude other disorders. In some cases there are findings in magnetic resonance imaging (MRI) on T2-weighted and FLAIR images that show cortical swelling and hyperintensity that are most likely caused by brain edema (Russell \& Ducros 2011, Bhatia \& Babtain 2011, Jacob et al. 2006).

Here we report a rare case of sporadic hemiplegic migraine with psychosis and brain edema on MRI.

\section{CASE REPORT}

We present a case of a 47-year-old Caucasian male with rare attacks (one or two times per year) of headache with migraine features preceded by visual impair- ment lasting up to one hour. He suffered from such headaches for the last 20 years. Headaches would last for three to four days and they were associated with mild confusion, disorientation and fever. The diagnostic work-up including two brain MRI scans, in 2002. and 2009., showed mild atrophy but otherwise were normal. Based on clinical findings and diagnostic exams he was diagnosed with migraine with aura. The patient did not take any prophylactic therapy. $\mathrm{He}$ has also been diagnosed with hypertrophic cardiomyopathy, prolapse of the mitral valve and anxiety depressive disorder.

In May 2017 the patient developed scintillating scotomas and bitemporal hemianopia followed within 30 minutes by strong headache and fever that lasted approximately 24 hours. After five days he developed dysarthria, paresthesia and paresis of the left extremities accompanied by headache. The whole time he had a mild confusion, he was disorientated and agitated. The patient was hospitalized and diagnostic work-up was performed: brain MRI showed cortical and subcortical edema of the right temporal, parietal and occipital lobe and edema of the right temporo-parietal operculum and insula on T2-weighted and FLAIR images (Figure 1). CSF analysis was normal except elevated lactates. In the next three days, complete improvement in condition and withdrawal of all symptoms was observed spontaneously. Follow-up was continued in the outpatient clinic.

Two months after his first symptoms appeared, a control MRI scan was performed which showed a mild regression of edema.

In August 2017, three months after the initial presentation, he suddenly experienced similar symptoms, predominantly cortical blindness and mild left hemiparesis accompanied by headache with migraine characteristics. In the next two days his mental state deteriorated with signs of aggression, elements of psychosis, suicidality and a coma like state. The treatment with antipsychotic drugs (haloperidol, quetiapine, promazine, clozapine) was started but without any improvement. 

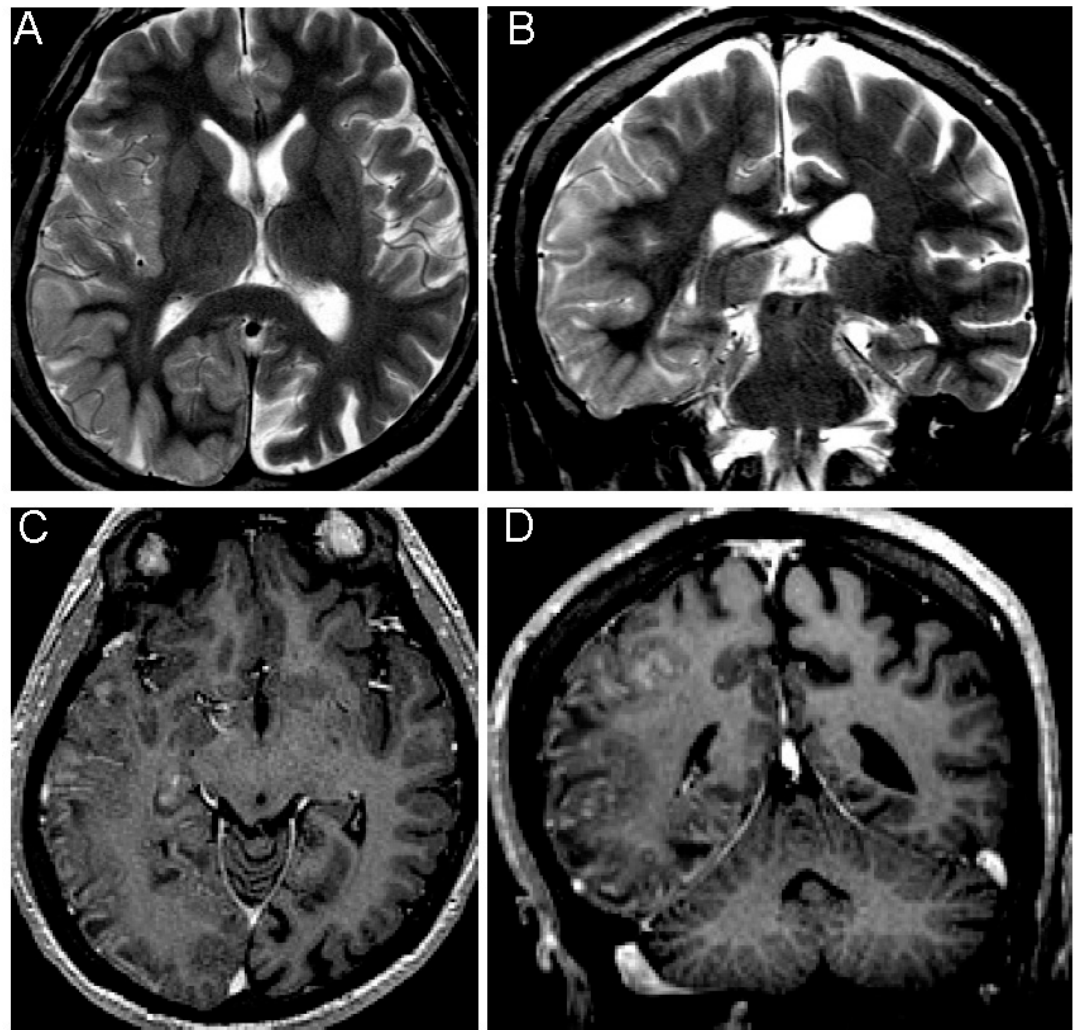

Figure 1. Initial brain MRI: T2-weighted (A,B) sequences showing hyperintensity and swelling of the grey matter in the right frontal, parietal, temporal and occipital regions. T1-weighted images with gadolinium $(\mathrm{C}, \mathrm{D})$ showing unilateral gyriform enhancement that corresponds to the regions of cortical edema

Lumbar puncture was repeated for several times, every time CSF analysis, including lactates, was normal. EEG showed diffuse slowing with elements of polymorphic delta activity. He was tested for viruses (HIV, HSV, HCV and HBV) and syphilis and the results came negative. The spectrum of antibodies for autoimmune encephalitis was made (LGI1, CASPR2, NMDAR, AMPAR, GABAR, anti-GAD, anti-Hu, antiYo, anti-Ri, anti-Ma2, anti-Tr, anti-amphiphysin) as were the tumor markers and all were within the reference interval. There was no evident tumor on the computed tomography (CT) of thorax, abdomen and pelvis. This lowered the suspicion that patient had some form of paraneoplastic or autoimmune encephalitis. The assessment for prion disease was made, 14-33 protein was negative and EEG did not show any activity that would encourage the diagnosis of Creutzfeldt-Jakob disease. All the immunology tests, genetic analysis on MELAS, MERRF, NARP and skin and muscle biopsy were negative. PET CT showed diffuse pathological hypometabolism of the brain parenchyma. MRI was made every week for the follow up and on the $27^{\text {th }}$ day of hospitalization, MRI showed a slight resolution of edema on T2-weighted and FLAIR images in the right hemisphere but there was a new region of edema in left parietal, temporal and occipital region and the enhancement was also seen on T1-weighted images with gadolinium (Figure 2). The suspicion arose that the changes seen on MRI examination could be part of sporadic hemiplegic migraine, especially in consideration with the patient's anamnesis.

Since his condition did not improve on current therapy with antipsychotic drugs, and also worsened because of severe pneumonia, the patient received five doses of intravenous immunoglobulins $(0.4 \mathrm{~g} / \mathrm{kg})$. He started to recover and the dosage of antipsychotic drugs was reduced. When his condition stabilized, ultrasound and CT of the heart were made and showed that he had spongiform cardiomyopathy. To prevent possible cardio embolic incidents, dabigatran was introduced (150 mg twice a day), as well as prophylaxis for migraine (valproate $500 \mathrm{mg}$ and propranolol $40 \mathrm{mg}$ twice a day). For prevention of psychiatric symptoms a small dosage of clozapine ( $6.25 \mathrm{mg}$ in the evening) was continued.

Two months after his discharge from hospital he is amnestic for the events during his illness. He is without any motor symptoms but he is complaining of mild dysfunction of peripheral vision. The treatment with propranolol and valproate was continued as before and all antipsychotic drugs were excluded from his treatment. His MRI scan showed slight gyral gliosis in the right frontal, parietal, temporal and occipital regions with atrophy of the right hippocampus on $\mathrm{T} 2$-weighted images and no residual enhancement was seen on T1-weighted images with gadolinium (Figure 3). Genetic testing for FHM came negative. 

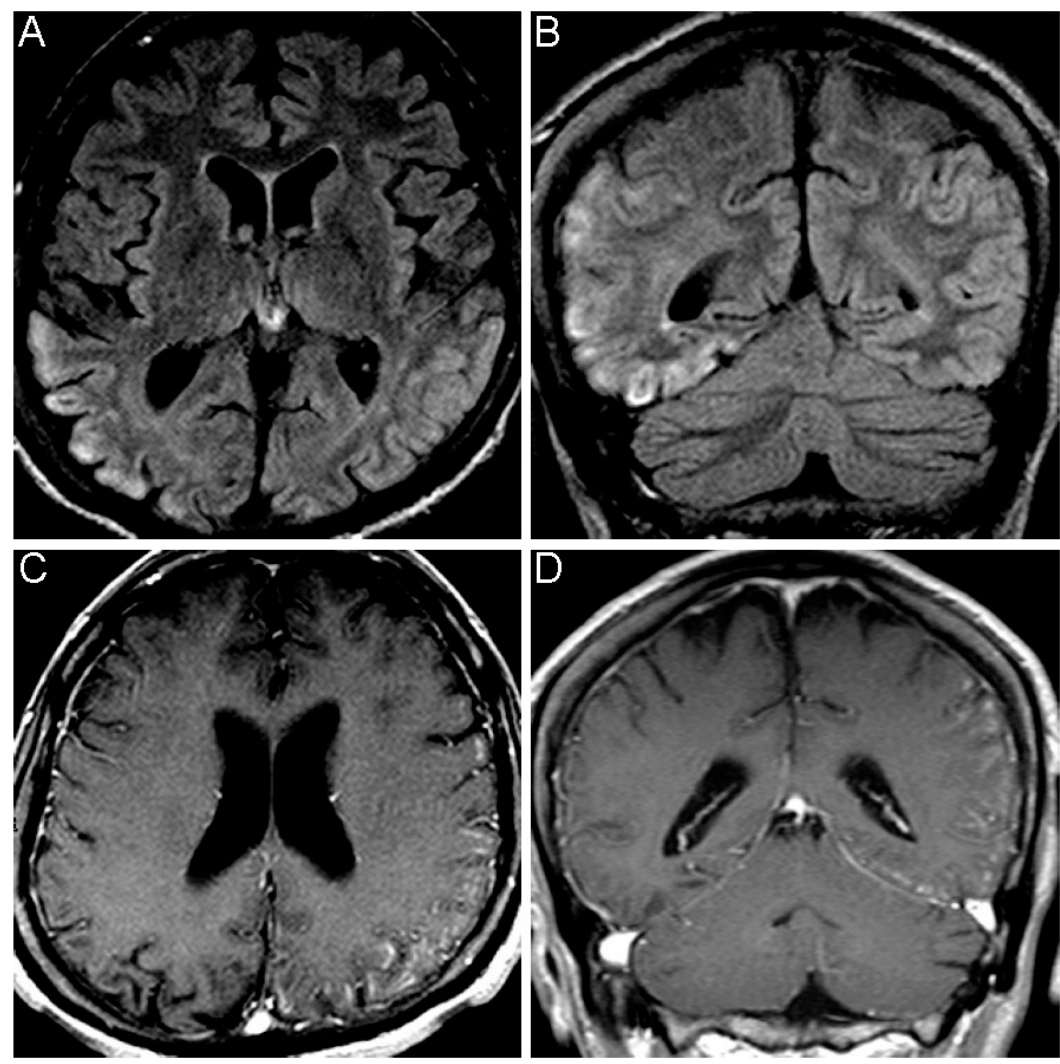

Figure 2. Brain MRI on day 27: partial resolution of cortical edema of the right cerebral hemisphere and a new region of mild cortical edema in left parietal, temporal and occipital regions on axial (A) and coronal (B) FLAIR sequences. T1weighted images with gadolinium $(\mathrm{C}, \mathrm{D})$ showed resolution of enhancement on the right hemisphere but there were new lesions on the left hemisphere in parietal, temporal and occipital areas that correspond to the regions of cortical edema
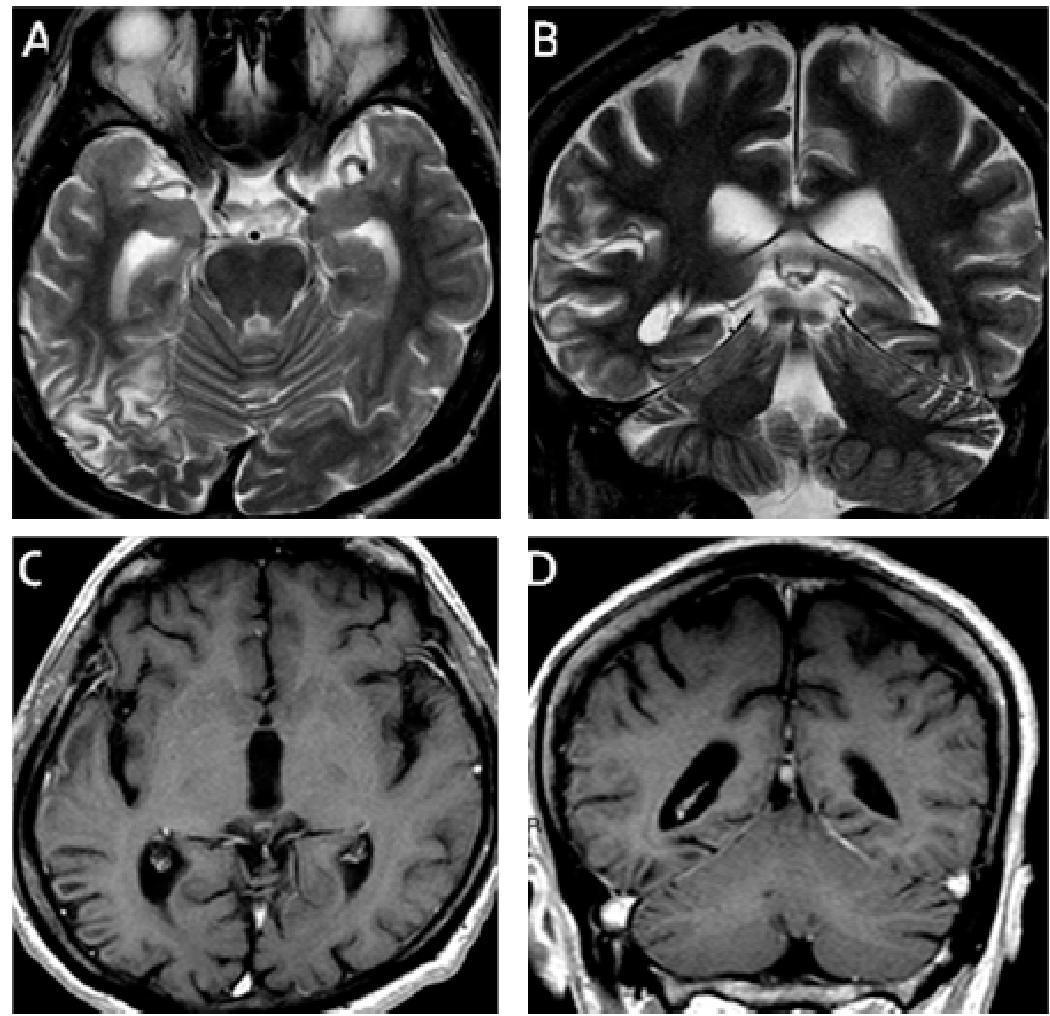

Figure 3. Control brain MRI after 2 months: T2-weighted axial (A) and coronal (B) images showing slight gyral gliosis in the right frontal, temporal and occipital regions with mild enlargement of temporal horn of the right lateral ventricle. T1-weighted images with gadolinium (C, D) showing no residual enhancment 


\section{DISCUSSION}

Sporadic hemiplegic migraine is a rare form of migraine with aura that can sometimes have a dramatic clinical picture with acute psychosis and impairment of consciousness.

In our case report, we presented a patient who suffered from migraine with aura and for the first time in 20 years he presented with clinical picture of headache followed by psychosis. Based on MRI appearance, the differential diagnosis could include stroke, encephalitis and gliomatosis cerebri (Bhatia \& Babtain 2011, Jacob et al. 2006). Cardioembolic stroke due to newly diagnosed spongiform cardiomyopathy was discarded because of the clinical presentation and MRI scan that strongly opposed an ischemic event as there was involvement of multiple arterial territories and absence of vascular occlusion.

Considering the extensive diagnostic evaluation that eliminated known autoimmune, paraneoplastic and infective causes of brain edema, we concluded that the clinical presentation and changes visible on neuroimaging were part of prolonged episode of SHM. In attempt to stabilize his psychological condition, haloperidol as the recommended first-line therapy for acute psychosis in SHM (Friedman et al. 2014) was introduced but in this case the efficacy of this drug was lacking.

As a last resort, treatment with intravenous immuneglobulins was tried even though there was no published data that immunoglobulins were ever used in SHM.

Pathophysiologic mechanisms in SHM and in other forms of migraine are not completely understood. It is proposed that the aura is caused by cortical spreading depression, a wave of neuronal and glial depolarization that spreads across the cerebral cortex (Tfelt-Hansen 2014, Ferrari et al. 2012). How to explain favorable effect of immunoglobulins in cortical spreading depression is unclear. A possible explanation for the immunoglobulin efficacy in this case maybe lies in a theory that psychosis is an autoimmune disorder (AlDiwani et al. 2017, Suvisaari \& Mantre 2013, Herken et al. 2017), which was the patient's main symptom. There is also a known positive effect of immunoglobulins in autoimmune encephalitis, where the psychiatric symptoms are one of the most pronounced features (Herken et al. 2017).

Brain atrophy with permanent cognitive impairment was described after episodes of hemiplegic migraine attacks and it was strongly associated with the duration of attacks (Pelzer et al. 2018). To prevent cognitive impairment, the emphasis should be on prevention of migraine attacks and also on rapid ending of this episodes.

\section{CONCLUSION}

We presented an unusual case of hemiplegic migraine with a prolonged confusional state; thorough diagnostic work-up has not revealed any linkable cause. Even though there is no evidence-based data for the use of immunoglobulins in the treatment of HM, it was successful in our patient. Due to the lack of knowledge about the pathophysiology of SHM, there is a need for further research to confirm the efficacy of immunoglobulin therapy in acute state of the disease and to find the most favorable profilactic therapy.

\section{Acknowledgements:}

We would like to thank the team from Danish Headache Center, Rigshospital - Glostrup, our neuroradiologist Assistant Professor David Ozretić and Profesor Fran Borovečki from Department for Functional Genomics, for their advices and guidance in this challenging case.

\section{Conflict of interest: None to declare.}

\section{Contribution of individual authors:}

Marina Mioč: research idea, study design, manuscript writing, literature search.

Vlatko Šulentić: manuscript writing, literature search, study design.

Ivan Jovanović: research idea, literature search, manuscript writing.

Vlasta Vuković Cvetković: literature search, manuscript writing.

Sibila Nanković \& Željka Petelin Gadže: manuscript revising.

\section{References}

1. Al-Diwani AAJ, Pollak TA, Irani SR, Lennox BR: Psychosis: an autoimmune disease? Immunology 2017; 152:388-401

2. Bhatia H, Babtain F: Sporadic Hemiplegic Migraine with Seizures and Transient MRI Abnormalities. Case Rep Neurol Med 2011; 2011:258372

3. Ferrari MD, Klever RR, Terwindt GM, et al.: Neuronal death by repetitive cortical spreading depression in juvenile rat brain. Exp Neurol 2012; 233:438-446

4. Friedman JI, Soleimani L, McGonigle DP, Egol C, Silverstein JH: Pharmalogical treatments of non-substancewithdrawal delirium: A systematic review of prospective trails. Am J Psychiatry 2014; 171:151-159

5. Headache Classification Committee of the International Headache Society (IHS): The International Classification of Headache Disorders, 3rd edition. Cephalalgia 2018; 38:1-211

6. Herken J, Pruss H. Red flags: Clinical signs for Identifyin Autoimmune Encephalits in Psychiatric Patients. Front Psychiatry 2017; 16:8-25

7. Jacob A, Mahavish K, Bowden A, Smith ET, Enevoldson $P$, White RP: Imaging abnormalities in sporadic hemiplegic migraine on conventional MRI, diffusion and perfusion MRI and MRS. Cephalalgia 2006; 26:1004-9 
8. LaBianca S, Jensen $R$, van den Maagdenberg AMJM, Baandrup L, Bendsten L: Familial Hemiplegic Migraine and Recurrent Episodes of Psychosis: A Case Report. Headache 2015; 55:1004-1007

9. Pelzer N, Hoogeeveen ES, Ferrai MD et al.: Brain atrophy following hemiplegic migraine attacks. Cephalalgia 2018; 38:1199-1202

10. Russell MB, Ducros A: Sporadic and familial hemiplegic migraine: pathophysiological mechanisms, clinical cha racteristics, diagnosis, and management. Lancet Neurol 2011; 10:457-70

11. Suvisaari J, Mantre O: Inflammation theories in psychotic disorders: a critical review. Infect Disord Drug Targets 2013; 13:59-70

12. Tfelt-Hansen PC: History of migraine with aura and cortical spreading depression from 1941 and onwards. Cephalgia 2010; 30:780-92

\section{Correspondence:}

Marina Mioč, MD

University Psychiatric Hospital Vrapče, University of Zagreb

Bolnička c. 32, 10090 Zagreb, Croatia

E-mail: marina.mioc29@gmail.com 